\title{
A Novel Multi-Proxy, Big Data Approach to Reconstructing Earth's Climate System Through Time
}

HAFIDA EL BILALI ${ }^{1}$, RICHARD E. ERNST ${ }^{2}$, TIMOTHY W. LYONS $^{3}$ AND CHARLES W. DIAMOND ${ }^{4}$

${ }^{1}$ Carleton University

${ }^{2}$ Tomsk State University

${ }^{3}$ University of California, Riverside

${ }^{4}$ University of California Riverside

Presenting Author: hafidaelbilali@cunet.carleton.ca

Earth's climate system is composed of many individual components, each one inextricably linked to the others through a multitude of feedbacks. However, when perturbations linked to first-order drivers are sufficiently strong, a tipping point is reached and crossed, leading to a cascade of positive feedbacks that ultimately settle into a new steady state. This process, which can be monitored through variations in geochemical proxies that track paleoclimatic and paleoceanic conditions, can be visualized as a topology ('landscape') of data 'basins' maintained by negative feedbacks along with 'hills/ridges' that represent tipping points. As a test of this approach to exploring Earth's ancient climate, we have produced a 'landscape' via a graphical approach using roughly 15,000 published carbon $\left(\delta^{13} \mathrm{C}\right)$ and oxygen $\left(\delta^{18} \mathrm{O}\right)$ isotope data from sedimentary carbonates spanning the past 2.5 billion years. While recognizing the likelihood of frequent resetting of carbonate-hosted proxy data during diagenesis, stability basins observed may represent negative feedback loops, and each major excursion between basins results from positive feedback loops often set into motion by external inputs that can overwhelm the system. We recognize multiple major stability basins/troughs and ridges/hills in Earth's climate system. We superimpose isotopic excursions and convergence of four subsets and infer that the values at the convergence must fingerprint the isotopic composition of the major drivers (likely primary and secondary) that caused the excursions. Our methodology can be applied to other large proxy datasets, and these multivariate statistical approaches can be used to develop a more robust and higher resolution 'landscape' of the climate system through Earth history-with applications to the modern and future climate. 